\title{
Effective Treatment of an Unhealed Incision of a Diabetic Patient with Recombinant Human Epidermal Growth Factor
}

\section{WKR Wong*}

Division of Life Science, The Hong Kong University of Science and Technology, Clear Water Bay, Kowloon, Hong Kong, China

\begin{abstract}
A facile protocol, which involves topical application of recombinant human Epidermal Growth Factor (rhEGF), was previously developed by our group and shown to be effective in treating diabetic foot ulcers, when working in conjunction with debridement surgery. In this communication, using the same approach of rhEGF application, despite the absence of debridement, our findings demonstrated that the protocol worked equally well on a hard-to-heal wound, which remained unhealed subsequent to surgical removal of the great saphenous vein from the left thigh of a diabetic patient. After treatment with rhEGF for $10 \mathrm{~d}$, the wound was shown to heal readily and finally achieved complete healing. The success of rhEGF in the healing process is concluded to be attributable to both the fine quality and sufficient dosage of rhEGF.
\end{abstract}

Keywords: Recombinant human epidermal growth factor; Escherichia coli; Excretion; Hard-to heal wounds; Topical application; Diabetes mellitus

\section{Abbreviations}

DFU: Diabetic Foot Ulcers; rhEGF: Recombinant human Epidermal Growth Factor employed in this study; hEGF: Human Epidermal Growth Factor produced by other protocols; VT: Designation of the diabetic patient concerned in this study; CAD: Coronary Artery Disease; CABG: Coronary Artery Bypass Grafting; EVH: Endoscopic Vein Harvesting; SJS: Steven Johnson Syndrome

\section{Introduction}

Patients suffering from Diabetes mellitus are potentially afflicted with many health- threatening complications. Among them, the presence of diabetic neuropathy and peripheral vascular damage may lead to the development of Diabetic Foot Ulcers (DFU), which results in a 5- to 50- fold higher risk of non-traumatic amputation when compared with normal individuals [1]. On the other hand, vascular damage causes coronary atherosclerosis, which may lead to myocardial infarction. Since good blood circulation is important for the healing of existing wounds and prevention of new wound development [2], cardiovascular disease has an undesirable effect on wound healing.

My group has pioneered the application of recombinant human Epidermal Growth Factor (rhEGF) to the treatment of DFU and complex wounds. In the early 2000s, employing rhEGF prepared from an engineered Escherichia coli excretion system [3-6], and working with a local hospital, United Christian Hospital, in Hong Kong, we reported the use of rhEGF to successfully enhance the healing rate of DFU [7]. In our protocol, rhEGF resuspended in aqueous cream was demonstrated to be able to dramatically improve the efficacy of complete recovery of DFU wounds. Specifically, it improved from a rate of $42.1 \%$ obtained using only debridement surgery to a much increased efficiency of $95 \%$, achieved through first debridement and subsequently topical treatment with $0.04 \%$ (w/w) rhEGF, but not a lower concentration of rhEGF with $0.02 \%(w / w)$ of it [7]. Subsequently, patent protection has also been obtained for the findings reported in this facile treatment process [812].

In addition to treating DFU [7], 0.04\% (w/w) rhEGF has also been applied successfully to cure other severe wounds, including Steven Johnson Syndrome [13] and bedsores [8-12]. All of these applications support the view that rhEGF is not only an effective means for wound healing, but it may be highly versatile in managing broadly different hard-to-heal wounds. In this communication, the application of rhEGF to an effective treatment of a recalcitrant wound, which resulted from the surgical removal of the great saphenous vein of the left thigh of a diabetic patient, is reported. Prior to the curing process, the wound suffered bacterial infections, thus rendering the chance of potential gangrene, which might lead to life-threatening conditions if delays in treatment occurred.

\section{Results and Discussion}

The patient was a 64-year-old male, designated "VT", who had suffered from Type 2 diabetes mellitus for 7 years. By taking prescribed drugs, metformin and glyburide, VT's blood sugar readings varied between 6 and $10 \mathrm{mmol} \mathrm{l}^{-1}$ before he was diagnosed with Coronary Artery Disease (CAD). To cure this potentially fatal heart disease, VT, who had never received any cardiac surgery previously, was recommended to undertake the treatment of Coronary Artery Bypass Grafting (CABG), in which three coronary arteries were required to be bypassed during surgery. To attain three vessels required in CABG, a 48 $\mathrm{cm}$ great saphenous vein was harvested from VT's left thigh (Figure 1), using the endoscopic method [14], and then employed for the surgical bypass. As a result of operations, four incisions, spreading from the groin to the knee and with each wound measuring approximately 4-6 $\mathrm{cm} \times 0.8 \mathrm{~cm}$, existed on his left thigh (Figure 1).

The CABG surgery progressed quite favorably. VT recovered swiftly from CAD after treatment and was discharged from the hospital $4 \mathrm{~d}$ after the surgery. At that point, despite VT's diabetic complications, all four incision wounds located on his left thigh appeared to heal well.

However, probably due to movements of the left leg, in about the $3^{\text {rd }}$ week or so after VT returned home from the hospital, the $2^{\text {nd }}$ wound from the knee (Figure 1) ruptured. While recovery of the remaining

*Corresponding author: Wong WKR, Division of Life Science, The Hong Kong University of Science and Technology, Clear Water Bay, Kowloon, Hong Kong, China, Tel: +852-235-872-99; Fax: +852-235-815-52; E-mail: bcwkrw@ust.hk

Received August 25, 2015; Accepted September 09, 2015; Published September 23, 2015

Citation: Wong WKR (2015) Effective Treatment of an Unhealed Incision of a Diabetic Patient with Recombinant Human Epidermal Growth Factor. Mod Chem appl 3: 166. doi:10.4172/2329-6798.1000166

Copyright: (c) 2015 Wong WKR, et al. This is an open-access article distributed under the terms of the Creative Commons Attribution License, which permits unrestricted use, distribution, and reproduction in any medium, provided the original author and source are credited. 
three wounds progressed quite well, the $2^{\text {nd }}$ wound did not exhibit any improvement in healing. In another 4 weeks, while the $1^{\text {st }}, 3^{\text {rd }}$ and $4^{\text {th }}$ wounds had almost completely healed, the $2^{\text {nd }}$ wound remained not only unhealed, but also developed small sloughs, despite being treated with cloxacillin, an antibiotic prescribed by VT's family doctor for him to take to prevent bacterial infections.

Despite taking cloxacillin and being monitored by a doctor, the $2^{\text {nd }}$ wound on VT's left thigh refused to heal (Figure 2). It appeared that further delays in treating the wound might induce life-threatening ulceration, which might lead finally to leg amputation. VT and his family were apprehensive, and they approached us for assistance seven weeks after Endoscopic Vein Harvesting (EVH).

With our previous experience and success in healing DFU, a facile protocol (Figure 3) was promptly designed to treat VT's unhealed $2^{\text {nd }}$ wound on his left thigh [7]. As in treating DFU, a simple topical procedure was proposed to be employed for the unhealed wound (Figure 3). However, unlike DFU, VT's wound was relatively "clean", i.e., it appeared to be only mildly infected. Moreover, there was not much dead tissue in or around the wound, thus precluding the requirement of a debridement procedure for wound cleansing. VT's wound was treated twice a day with an aqueous cream containing $0.04 \%$ (w/w) rhEGF (Figure 3 ), which was previously utilized successfully in treating DFU [7], bedsores [8-12] and SJS [13]. The result was unexpectedly positive. VT's wound showed efficient recovery, and thus rhEGF application was stopped after the $10^{\text {th }}$ day of treatment. Over approximately $6 \mathrm{~d}$, despite the absence of rhEGF treatment, VT's wound continued to recover and subsequently achieved complete healing (Figure 2). Another point worth noting is that the wound healed not only efficiently, but apparently also quite smoothly, thus avoiding the formation of a hypertrophic scar on the skin (Figure 2).

Previously, we reported that rhEGF was employed successfully in treating various complex wounds including DFU [7], bedsores [8-12], and SJS [13]. In the current study, it was again well demonstrated that rhEGF was effective in promoting treatment of a seriously deteriorating wound of a diabetic patient (Figure 1) which refused to heal seven weeks after the surgical process. The high efficacy of rhEGF in wound healing was likely attributable to two important factors, firstly, to the high concentration, $0.04 \%$ (w/w) rhEGF, which was revealed to be above the minimum effective dosage in treating DFU [7]. Secondly, the fine quality of rhEGF might also play a crucial role. The excretion system engineered by our group has been utilized to efficiently produce rhEGF, which has been shown to possess not only potent bioactivity, but also exceptional purity [5]. Both the high levels of bioactivity and purity of rhEGF were concluded to have critical impact on curing the recalcitrant wound on VT's thigh (Figure 2).

Given the beneficial applications of hEGF in treating various wounds, the possibility that hEGF, which has a potent proliferative effect on skin cells, might stimulate malignancy has always constituted a subject of concern. However, vast amounts of data have been gathered about applications of hEGF in treating a wide variety of wounds through various routes of administration [15]. Interestingly, despite the reports of numerous successful treatments in these studies, none of them has offered significant evidence to support that exogenous introduction of hEGF would promote carcinogenesis $[15,16]$. On the other hand, mutants of EGF receptor, which are able to undertake ligand-independent gene activation and possess increased ligand affinity, have been speculated to be a cause of malignant transformation [16]. In yet another direction, it may be interesting to investigate the ability of different forms of hEGF, including authentic hEGF and its

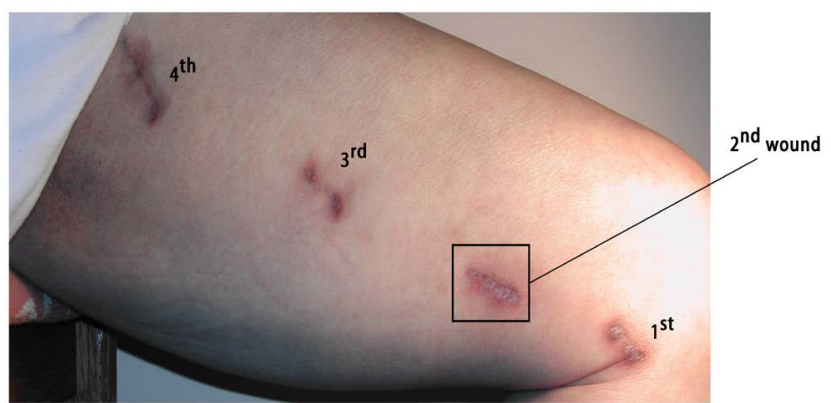

Figure 1: Four incisions resulting from endoscopic harvesting of the great saphenous vein from VT's left thigh. Labels $1^{\text {st }}$ to $4^{\text {th }}$ indicate positions of the four incisions. The $2^{\text {nd }}$ wound (boxed) refused to heal and was then subjected to treatment with $0.04 \%(\mathrm{w} / \mathrm{w})$ rhEGF.

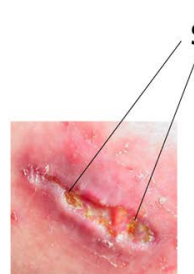

Day 0

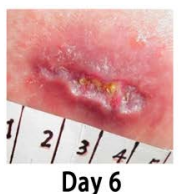

Day 6

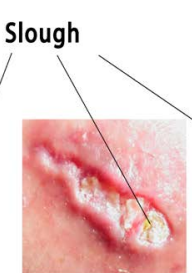

Day 2

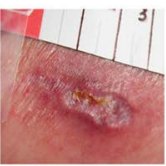

Day 10

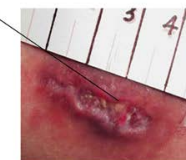

Day 4

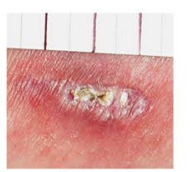

Day 16

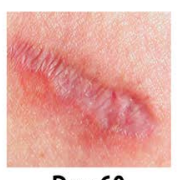

Day 60
Figure 2: Healing of the unhealed wound $\left(2^{\text {nd }}\right.$ one shown in Figure 1) treated with $0.04 \%(w / w)$ rhEGF. Day 0 to Day 60 stand for the stages of healing of the wound at various times: Day 0 (before treatment); Day 2 to Day 10 (treatment with rhEGF for 2-10 d). The treatment was stopped after Day 10; Day 16 and Day 60 reveal the progress of healing of the wound on Day 16 and Day 60, respectively. Slough symptoms in the wound are indicated.

\section{Wound}

(1) Cleanse with diluted hydrogen peroxide or dettol

(2) Air dry

(3) Topically apply a slight film of rhEGF

(4) Place a gauze pad on top

(5) Monitor progress of healing; repeat (1) to (4) if necessary

\section{Complete healing}

Figure 3: A facile protocol for treating the unhealed wound $\left(2^{\text {nd }}\right.$ one shown in Figure 1). Five simple steps (1-5) are involved in the protocol. In step (1), the dilutions are performed according to the manufacturer's recommendations. In step (3), the $0.04 \%$ (w/w) rhEGF film should cover the wound completely. In step (4), the gauze pad is taped to fix its position. 
derivatives, in stimulating malignancy. Since rhEGF shares the same identity as native hEGF [5], it is believed that the former will be as safe as the latter in promoting cell growth.

A wide range of expression systems have been developed for the production of hEGF $[17,18]$, which comprised a collection of structurally heterogeneous products $[17,18]$. In fact, many of these systems were able to produce only structural derivatives of mature native hEGF $[17,18]$, which might not be as active or stable as rhEGF [3-6]. The later product was characterized to possess an authentic structure, thus sharing the same primary sequence as mature native hEGF of human origin [5]. Moreover, hEGF produced by the majority of the protocols, which were designed essentially for intracellular protein production, were likely contaminated by endogenous proteins and/or endotoxins of the host cells. These contaminants could seriously impede the bioactivity and stability of hEGF. For example, it has been asserted that many of these protocols have resulted in even non-active hEGF [19].

\section{Conclusion}

CAD, similar to DFU, is a common side effect of diabetes. CABG is often considered a solution to treat $\mathrm{CAD}$. When an incision resulting from saphenous venous harvesting refused to heal, gangrene may develop and lead to life-threatening complications. In this report, as demonstrated previously by our group in treating DFU, topical treatment with $0.04 \%(\mathrm{w} / \mathrm{w})$ rhEGF is shown to be equally effective in promoting healing of a hard-to-heal surgical wound derived from EVH.

\section{Acknowledgements}

Recombinant human Epidermal Growth Factor (rhEGF) was a generous gift from Gene-vinate Limited, a member of the HKUST Entrepreneurship Program.

\section{References}

1. Most RS, Sinnock P (1983) The epidemiology of lower extremity amputations in diabetic individuals. Diabetes Care 6: 87-91.

2. Jordan S (2015) Why good blood circulation is important? The Dallas Weekly: Health.

3. Wong DKH, Lam KHE, Chan CKP, Wong YCV, Wong WKR, et al. (1998) Extracellular expression of human epidermal growth factor encoded by an Escherichia coli K-12 plasmid stabilized by the ytl2-incR system system of Salmonella typhimurium. J Ind Microbiol Biotechnol 21: 31-36.

4. Sivakesava S, Xu ZN, Chen YH, Hackett J, Huang RC, et al. (1999) Production of excreted human epidermal growth factor (hEGF) by an efficient recombinant Escherichia coli system. Process Biochem 34: 893-900.
5. Huang RC, Lam E, Chen YH, Hackett J, Lam TL, et al (1999) Human epidermal growth factor excreted by recombinant Escherichia coli K-12 has the correct $\mathrm{N}$-terminus and is fully bioactive. Process Biochem 35: 1-5

6. Wong WKR, Fu BZB, Wang YY, Ng AKL, Chan AKN (2012) Engineering of efficient Escherichia coli excretion systems for the production of heterologous proteins for commercial applications. Recent Patents on Chemical Engineering 5: 45-55.

7. Tsang MW, Wong WKR, Hung CS, Lai KM, Tang W, et al. (2003) Human epidermal growth factor enhances healing of diabetic foot ulcers. Diabetes Care 26: 1856-1861.

8. Wong WKR, Lam KHE, Tsang MW (2014) Method and composition for treating skin wounds with epidermal growth factor. PCT European Patent EP1482966B1.

9. Wong WKR, Lam KHE, Tsang MW (2014) Method and composition for treating skin wounds with epidermal growth factor. Hong Kong Patent HK1071525.

10. Wong WKR, Lam KHE, Tsang MW (2011) Method and composition for treating skin wounds with epidermal growth factor. Taiwan PCT National Phase Patent Taiwanese Patent No.: I 348912.

11. Wong WKR, Lam KHE, Tsang MW. (2009) Method and composition for treating skin wounds with epidermal growth factor. China PCT National Phase Patent, Chinese Patent No. ZL03809985.3.

12. Wong WKR, Lam KHE, Tsang MW (2009) Method and composition for treating skin wounds with epidermal growth factor. United States PCT National Phase Patent, US Patent No. 7,517,528 B2.

13. Tsang MW, Tsang KY, Wong WKR (2004) A case report on the use of recombinant human epidermal growth factor (rhEGF) in a gentlemen with drug induced Steven Johnson Syndrome. Derm Online J 10: 25.

14. Raja SG, Sarang Z (2013) Endoscopic vein harvesting: technique, outcomes, concerns \& controversies. J Thorac Dis 5: S630-S637.

15. Berlanga-Acosta J, Gavilondo-Cowley J, López-Saura P, González-López T, Castro-Santana MD, et al. (2009) Epidermal growth factor in clinical practice - a review of its biological actions, clinical indications and safety implications. Int Wound J 6: 331-346.

16. Berlanga-Acosta J, Gavilondo-Cowley J, del Barco-Herrera DG, MartínMachado J, Guillen-Nieto G (2011) Epidermal Growth Factor (EGF) and Platelet- Derived Growth Factor (PDGF) as Tissue Healing Agents: Clarifying Concerns about their Possible Role in Malignant Transformation and Tumor Progression. J Carcinogene Mutagene 1: 115.

17. Ferrer Soler L, Cedano J, Querol E, de Llorens R (2003) Cloning, expression and purification of human epidermal growth factor using different expression systems. J Chromatogr B Analyt Technol Biomed Life Sci 788: 113-123.

18. Yadwad VB, Wilson S, Ward OP (1994) Production of human epidermal growth factor by an ampicillin resistant recombinant Escherichia coli strain. Biotechno Lett 16: 885-890.

19. Razis AFA, Ismail EN, Hambali Z, Abdullah MNH, Ali AM, et al. (2006) The Periplasmic Expression of Recombinant Human Epidermal Growth Factor (hEGF) in Escherichia coli. Asia Pac J Mol Biol Biotechnol 14: 41-45. 